\title{
Azimuthal asymmetries as the probe of nuclear matter at EIC
}

\section{Yu-kun Song*}

School of Physics and Technology, University of Jinan, Jinan, Shandong 250022, China

E-mail: sps_songyk@ujn.edu.cn

\begin{abstract}
We study the nuclear dependence of transverse-momentum-dependent (TMD) parton distribution functions (PDFs) and azimuthal asymmetries in semi-inclusive DIS off polarized nuclear targets. Multiple gluon interactions which generate gauge links in the operator definitions of PDFs also induce nuclear effects. The general Gaussian-type broadening effects on TMD PDFs suppress azimuthal asymmetries for eA SIDIS relative to that of eN case, with suppression factors expressed by the jet transport coefficient $\hat{q}$. We study nuclear effects on various azimuthal asymmetries, and calculate the suppression factor with $\hat{q}$ extracted from literature. The numeric results will be helpful for the experimental study of nuclear partonic structure from Semi-inclusive DIS at future EIC.
\end{abstract}

QCD Evolution 2016

May 30-June 03, 2016

National Institute for Subatomic Physics (Nikhef) in Amsterdam

\footnotetext{
${ }^{*}$ Speaker.
} 


\section{Azimuthal asymmetris of semi-inclusive DIS}

Semi-inclusive deeply inelastic scattering (SIDIS) provides a good place to study partonic structure of nucleon/nucleus, especially the transverse-momentum-dependent parton distribution functions (TMD PDFs). Azimuthal asymmetries of semi-inclusive DIS are nice probes to various TMD PDFs with defferent spin and transverse momentum correlations. We have employed collinear expansion $[1,2,3]$ to calculate the cross section and azimuthal asymmetries of semiinclusive DIS $[4,5,6]$. For the process $e^{-}\left(l, s_{l}\right)+N(p, s) \rightarrow e^{-}\left(l^{\prime}\right)+q(k)+X$, the differential cross section reads,

$$
\frac{d \sigma}{d x_{B} d y d^{2} k_{\perp}}=\frac{2 \pi \alpha_{\mathrm{em}}^{2} e_{q}^{2}}{Q^{2} y}\left(W_{U U}+\lambda_{l} W_{L U}+s_{\perp} W_{U T}+\lambda W_{U L}+\lambda_{l} \lambda W_{L L}+\lambda_{l} s_{\perp} W_{L T}\right),
$$

where $x_{B}$ the Bjorken variable, $y=\frac{p \cdot q}{p \cdot k}$ and $Q^{2}=-q^{2}=-\left(l-l^{\prime}\right)^{2}$. The structure functions $W_{s_{l} s}$ 's represent contributions in different polarization cases with $U, L, T$ standing for unpolarized, longitudinal-polarized and transversely-polarized beam/target. Each structure function contain contributions from different TMD PDFs. For example,

$$
\begin{aligned}
W_{U U} & =A(y) f_{1}\left(x_{B}, k_{\perp}\right)-\frac{2 x_{B}\left|\vec{k}_{\perp}\right|}{Q} B(y) f^{\perp}\left(x, k_{\perp}\right) \cos \phi, \\
W_{L U} & =-\frac{2 x_{B}\left|\vec{k}_{\perp}\right|}{Q} D(y) g^{\perp}\left(x_{B}, k_{\perp}\right) \sin \phi, \\
W_{U L} & =-\frac{2 x_{B}\left|\vec{k}_{\perp}\right|}{Q} B(y) f_{L}^{\perp}\left(x_{B}, k_{\perp}\right) \sin \phi,
\end{aligned}
$$

where $A(y)=1+(1-y)^{2}, B(y)=2(2-y) \sqrt{1-y}$ and $D(y)=2 y \sqrt{1-y}$. For other structure functions see [6]. The TMD PDFs involved has the following operator definitions,

$$
\begin{aligned}
f_{1}\left(x, k_{\perp}\right) & =\int \frac{d y^{-}}{4 \pi} e^{i x p^{+} y^{-}-i \vec{k}_{\perp} \cdot \vec{y}_{\perp}}\left\langle N\left|\bar{\psi}(0) \gamma^{+} \mathscr{L}(0 ; y) \psi(y)\right| N\right\rangle, \\
f^{\perp}\left(x, k_{\perp}\right) & =\frac{1}{k_{\perp}^{2}} \int \frac{p^{+} d y^{-}}{4 \pi} e^{i x p^{+} y^{-}-i \vec{k}_{\perp} \cdot \vec{y}_{\perp}}\left\langle N\left|\bar{\psi}(0) k_{\perp} \mathscr{L}(0 ; y) \psi(y)\right| N\right\rangle, \\
f_{L}^{\perp}\left(x, k_{\perp}\right) & =\frac{1}{k_{\perp}^{2}} \int \frac{p^{+} d y^{-}}{4 \pi} e^{i x p^{+} y^{-}-i \vec{k}_{\perp} \cdot \vec{y}_{\perp}}\left\langle N,+\left|\bar{\psi}(0) \varepsilon_{\perp i j} k_{\perp}^{i} \gamma_{\perp}^{j} \mathscr{L}(0 ; y) \psi(y)\right| N,+\right\rangle, \\
g^{\perp}\left(x, k_{\perp}\right) & =\frac{1}{k_{\perp}^{2}} \int \frac{p^{+} d y^{-}}{4 \pi} e^{i x p^{+} y^{-}-i \vec{k}_{\perp} \cdot \vec{y}_{\perp}}\left\langle N,+\left|\bar{\psi}(0) \varepsilon_{\perp i j} k_{\perp}^{i} \gamma_{\perp}^{j} \gamma_{5} \mathscr{L}(0 ; y) \psi(y)\right| N,+\right\rangle,
\end{aligned}
$$

where $\mathscr{L}(0 ; y)$ is generated by multiple gluon interactions of semi-inclusive DIS, and the existence of $\mathscr{L}(0 ; y)$ gurantee the gauge invariance of the operator definitions.

With cross section for semi-inclusive DIS, we can obtain various azimuthal asymmetries. For example,

$$
\begin{aligned}
& \langle\sin \phi\rangle_{L U}=-\lambda_{l} \frac{\left|\vec{k}_{\perp}\right|}{Q} \frac{D(y)}{A(y)} \frac{x_{B} g^{\perp}\left(x_{B}, k_{\perp}\right)}{f_{1}\left(x_{B}, k_{\perp}\right)}, \\
& \langle\sin \phi\rangle_{U L}=-\lambda \frac{\left|\vec{k}_{\perp}\right|}{Q} \frac{B(y)}{A(y)} \frac{x_{B} f_{L}^{\perp}\left(x_{B}, k_{\perp}\right)}{f_{1}\left(x_{B}, k_{\perp}\right)}
\end{aligned}
$$


By measuring these azimuthal asymmetries we can study various corresponding TMD PDFs. The factor $\left|\vec{k}_{\perp}\right| / Q$ shows that above 2 asymmetries come from twist- 3 contributions. Above formulae work equally well for both nucleon and nucleus target.

\section{Nulcear dependences}

The TMD PDFs contain the information of multiple gluon interactions between the struck quark and the remnant of the target. Gauge link can be naturally derived by this method wth correct direction to $+\infty$ for SIDIS and $-\infty$ for Drell-Yan. Multiple gluon interactions also lead to nuclear $k_{T}$-broadening of TMD quark distributions. For a loosely bounded large nucleus, the quark-quark correlation matrix,

$$
\Phi_{\alpha}^{A}\left(x, k_{\perp}\right) \equiv \int \frac{p^{+} d y^{-} d^{2} y_{\perp}}{(2 \pi)^{3}} e^{i x p^{+} y^{-}-i \vec{k}_{\perp} \cdot \vec{y}_{\perp}}\left\langle A\left|\bar{\psi}(0) \Gamma_{\alpha} \mathscr{L}(0 ; y) \psi(y)\right| A\right\rangle,
$$

has a Gaussian broadening with respect to nucleon [7, 8],

$$
\Phi_{\alpha}^{A}\left(x, k_{\perp}\right) \approx \frac{A}{\pi \Delta_{2 F}} \int d^{2} \ell_{\perp} e^{-\left(\vec{k}_{\perp}-\vec{\ell}_{\perp}\right)^{2} / \Delta_{2 F}} \Phi_{\alpha}^{N}\left(x, \ell_{\perp}\right),
$$

where $\Delta_{2 F}=\int d \xi_{N}^{-} \hat{q}_{F}\left(\xi_{N}^{-}\right)$is just a line integral of the quark transport coefficient of the nucleus medium. We can extract the nuclear broadening of nuclear TMD PDFs [9], for example,

$$
\begin{aligned}
f_{1}^{A}\left(x, k_{\perp}\right) & \approx \frac{A}{\pi \Delta_{2 F}} \int d^{2} \ell_{\perp} e^{-\left(\vec{k}_{\perp}-\vec{\ell}_{\perp}\right)^{2} / \Delta_{2 F}} f_{1}^{N}\left(x, \ell_{\perp}\right), \\
k_{\perp}^{2} g^{\perp A}\left(x, k_{\perp}\right) & \approx \frac{A}{\pi \Delta_{2 F}} \int d^{2} \ell_{\perp} e^{-\left(\vec{k}_{\perp}-\vec{\ell}_{\perp}\right)^{2} / \Delta_{2 F}}\left(k_{\perp} \cdot \ell_{\perp}\right) g^{\perp N}\left(x, \ell_{\perp}\right), \\
k_{\perp}^{2} f_{L}^{\perp A}\left(x, k_{\perp}\right) & \approx \frac{2 J_{A}}{\pi \Delta_{2 F}} \int d^{2} \ell_{\perp} e^{-\left(\vec{k}_{\perp}-\vec{\ell}_{\perp}\right)^{2} / \Delta_{2 F}}\left(k_{\perp} \cdot \ell_{\perp}\right) f_{L}^{\perp N}\left(x, \ell_{\perp}\right),
\end{aligned}
$$

To estimate the numerical size we take Gaussian ansatz for $k_{T}$-distribution for and take identical width $\alpha$ for all TMD PDFs. The ratio of the azimuthal asymmetries of the nucleus/nucleon involved semi-inclusive DIS are thus given by,

$$
\begin{aligned}
\frac{\langle\cos \phi\rangle_{L U}^{e A}}{\langle\cos \phi\rangle_{L U}^{e N}} \approx \frac{\alpha}{\alpha+\Delta_{2 F}}, \\
\frac{\langle\cos \phi\rangle_{U L}^{e A}}{\langle\cos \phi\rangle_{U L}^{e N}} \approx \frac{2 J_{A}}{A} \frac{\alpha}{\alpha+\Delta_{2 F}},
\end{aligned}
$$

The factor $f_{s}=\frac{\alpha}{\alpha+\Delta_{2 F}}$ is key to the nuclear suppression, and we estimate its value by adopting $\alpha$ and $\hat{q}$ values from literature [10,11, 12]. The explicit dependence on $A$ is given by [9],

$$
f_{s} \approx \frac{1}{1+0.114 A^{1 / 3}}
$$

The plot of this formula and fit to $k_{T}$-broadening are shown in the following figure. 


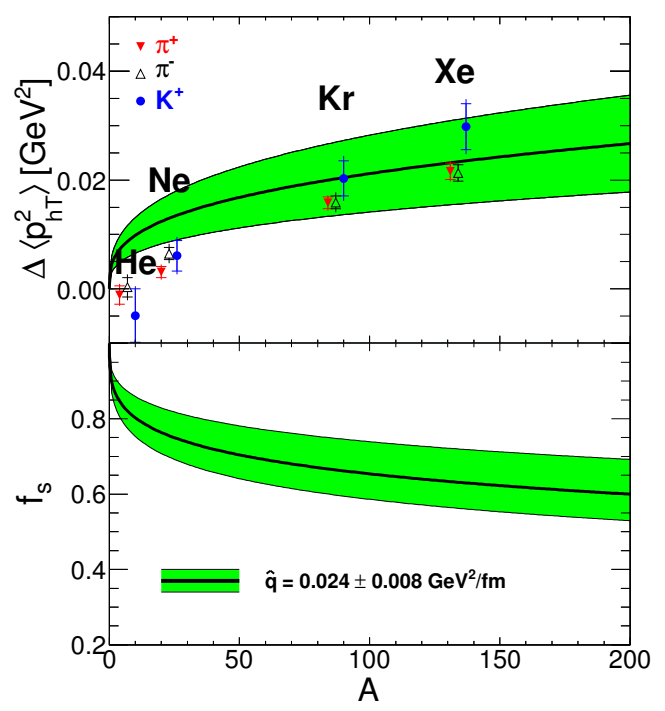

Figure 1: Nuclear $k_{T}$-broadening (upper panel) and nuclear suppression factor $f_{s}$ for azimuthal asymmetries [9].

\section{Conclusion}

We employ collinear expansion to study the azimuthal asymmetries and their nuclear dependences for semi-inclusive DIS. The Gaussian broadening effects generally suppress the asymmetries, and the suppression factor can be quantitatively estimated with reliable input for $k_{T}$ width and jet transport coefficient from literature. Azimuthal asymmetries show good potential as a new probe of the properties of nuclear medium. This will be a nice topic on future electron-ion collider (EIC).

\section{References}

[1] R. K. Ellis, W. Furmanski and R. Petronzio, Nucl. Phys. B 207 (1982) 1. doi:10.1016/0550-3213(82)90132-8

[2] R. K. Ellis, W. Furmanski and R. Petronzio, Nucl. Phys. B 212 (1983) 29. doi:10.1016/0550-3213(83)90597-7

[3] J. W. Qiu, Phys. Rev. D 42 (1990) 30. doi:10.1103/PhysRevD.42.30

[4] Z. t. Liang and X. N. Wang, Phys. Rev. D 75 (2007) 094002 doi:10.1103/PhysRevD.75.094002 [hep-ph/0609225].

[5] Y. k. Song, J. h. Gao, Z. t. Liang and X. N. Wang, Phys. Rev. D 83 (2011) 054010 doi:10.1103/PhysRevD.83.054010 [arXiv:1012.4179 [hep-ph]].

[6] Y. k. Song, J. h. Gao, Z. t. Liang and X. N. Wang, Phys. Rev. D 89 (2014) no.1, 014005 doi:10.1103/PhysRevD.89.014005 [arXiv:1308.1159 [hep-ph]].

[7] Z. t. Liang, X. N. Wang and J. Zhou, Phys. Rev. D 77 (2008) 125010 doi:10.1103/PhysRevD.77.125010 [arXiv:0801.0434 [hep-ph]]. 
[8] J. H. Gao, Z. t. Liang and X. N. Wang, Phys. Rev. C 81 (2010) 065211 doi:10.1103/PhysRevC.81.065211 [arXiv:1001.3146 [hep-ph]].

[9] Y. k. Song, Z. t. Liang and X. N. Wang, Phys. Rev. D 89 (2014) no.11, 117501 doi:10.1103/PhysRevD.89.117501 [arXiv:1402.3042 [nucl-th]].

[10] M. Anselmino, M. Boglione, U. D’Alesio, S. Melis, F. Murgia and A. Prokudin, Phys. Rev. D 88 (2013) no.5, 054023 doi:10.1103/PhysRevD.88.054023 [arXiv:1304.7691 [hep-ph]].

[11] W. t. Deng and X. N. Wang, Phys. Rev. C 81 (2010) 024902 doi:10.1103/PhysRevC.81.024902 [arXiv:0910.3403 [hep-ph]].

[12] N. B. Chang, W. T. Deng and X. N. Wang, Phys. Rev. C 89 (2014) no.3, 034911 doi:10.1103/PhysRevC.89.034911 [arXiv:1401.5109 [nucl-th]]. 